\title{
El ajuste por riesgo en la evaluación del desempeño hospitalario
}

\author{
Risk-adjustment in the assessment of hospital performance
}

\author{
Teddy Osmin Tamargo Barbeito'; Rosa Eugenia Jiménez Paneque" \\ 'Máster en Salud Pública. Especialista de I Grado en Bioestadística. Hospital Clínico \\ Quirúrgico "Hermanos Ameijeiras". La Habana, Cuba. \\ "Doctora en Ciencias Médicas. Especialista de II Grado en Bioestadística. Hospital \\ Clínico Quirúrgico "Hermanos Ameijeiras". La Habana, Cuba.
}

\section{RESUMEN}

Los indicadores de resultados constituyen parte esencial de la evaluación de la calidad de la atención hospitalaria. Son también atractivos pues permiten estimar el beneficio que representa el hospital para la comunidad, pero no son útiles para comparaciones en el espacio y el tiempo si no se ajustan por las características de los pacientes que fueron incluidos en el cálculo de los indicadores. La primera noción de esta necesidad proviene del siglo XIX y ya a mediados del siglo XX el ajuste por riesgo era una realidad en la comparación de desempeño hospitalario de países desarrollados. En Cuba aún no se realizan comparaciones de resultados en tiempo y espacio, dentro y entre hospitales o servicios hospitalarios, ajustadas por riesgo. Con el presente trabajo se pretende introducir el tema a los interesados y también abrir el debate en torno a la mejor forma de comenzar a realizar el ajuste por riesgo, imprescindible en las evaluaciones del desempeño hospitalario.

Palabras clave: Ajuste por riesgo, desempeño hospitalario, calidad.

\section{ABSTRACT}

Outcome indicators are an essential part of the hospital care quality assessment. They are also attractive elements that allow estimating the benefits that hospital brings to the community, but they are not useful in spatial and time comparisons if 
they are not adjusted to the characteristics of those patients included in the indicator calculations. The first notion of this requirement dated back to the 19th century, and at the middle of the 20th century, the risk-adjustment had already been included in the comparison of hospital performances in the developed countries. However, risk-adjusted spatial and time comparisons of outcomes inside the hospital or between hospitals or hospital services are not made in Cuba yet. The present paper attempted to submit this topic to the interested people and to pave the way for debate around the best form of introducing the risk-adjustment since it is essential for hospital performance assessments.

Key words: Risk adjustment, hospital performance, quality.

\section{NTRODUCCI ÓN}

La atención hospitalaria es el más caro de los servicios que se prestan en el área de la salud. El paciente en una unidad hospitalaria, además de ser atendido, desde el punto de vista médico, con la más alta tecnología, debe tener resueltas sus necesidades de alimentación y, en cierta forma, de vestuario, ${ }^{1}$ una realidad que justifica la necesidad de evaluar y controlar la eficiencia de la gestión hospitalaria. En términos más precisos, se conoce por ejemplo que en Cuba el recurso cama consume más del sesenta por ciento del presupuesto destinado a la salud. ${ }^{1,2}$ En otros países el presupuesto destinado a hospitales es también alto, por ejemplo, en Australia en los años 80 se informa que el presupuesto dedicado a hospitales constituyen el $43 \%$ del dedicado a la salud, Canadá en el 2004 reporta también 43 $\%$ del presupuesto dedicado a la salud, para gastos en hospitales y servicios médicos; en 1998 Suecia registra que el $62 \%$ de los gastos totales de los consejos regionales se dedican a la atención terciaria y secundaria altamente especializada; en el 2002, Hungría notifica que el $28 \%$ de sus gastos de salud se debe a gastos en servicios hospitalarios. ${ }^{3}$

Por otro lado, la eficiencia se considera parte de la calidad y ambas permiten evaluar el llamado "desempeño hospitalario". ${ }^{4-6}$ Un buen control de este desempeño requiere indicadores que permitan una evaluación de manera continuada; es decir, cifras que puedan consultarse con una periodicidad estable y adecuada y no sujetas a los vaivenes de decisiones administrativas o políticas.

Los llamados "Indicadores de resultados", ${ }^{7}$ como las tasas de mortalidad, las de reingreso o las de complicaciones y también el promedio de estadía, son especialmente apropiados para este fin puesto que reflejan el resultado concreto de la atención hospitalaria en términos de salud alcanzada o su complemento. Pero, es conocido que los cambios que se producen en este tipo de indicadores no dependen solamente de la calidad y la eficiencia de la atención que se brinda, sino de las disímiles características de los pacientes que constituyen el sustrato para calcularlos. Por lo tanto, cualquier valoración sobre el desempeño hospitalario que se realice a partir de un indicador de resultados debe contemplar de alguna manera las características de los pacientes que se utilizaron para estimar su valor. ${ }^{8,9}$ Un antecedente histórico que ilustra esta necesidad fue la reacción hostil de muchos médicos cuando, en 1862, William Farr, un colaborador de Florence Nightingale, 
publicó las tasas de mortalidad de varios hospitales ingleses. ${ }^{10-12} \mathrm{Si}$ bien varias de las críticas a estas cifras de Farr, se dirigían a la manera en que calculaba las tasas (utilizando denominadores inapropiados desde el punto de vista moderno), otras hacían alusión a lo que hoy se llamaría falta de "ajuste de riesgo", por ejemplo, uno de los críticos consideraba las comparaciones entre hospitales rurales y urbanos como inútiles, literalmente decía:

Cualquier comparación que ignore la diferencia entre los trabajadores con cachetes de manzana de las granjas que buscan atención en Stoke Pogis, (probablemente por reumatismo y dolor de piernas), y los enjutos y "parecidos a arenques rojos" (sic) mecánicos de Soho o Southwark que vienen de un hospital de Londres, es una falacia... ${ }^{12}$

Es decir que, la comparación de los resultados de la atención médica entre hospitales, con intenciones evaluativas, no conduce a valoraciones justas si no controla las diferencias debidas a las características y los riesgos de los pacientes.

A ese control de las variables que reflejan las características de los pacientes, necesario para atribuir diferencias a problemas de atención, se le ha denominado ajuste de riesgo (en inglés, risk adjustment). Realizar ajuste de riesgo significa contemplar, en las comparaciones, las diferencias entre pacientes en cuanto al riesgo de alcanzar determinado resultado, que no dependen de la calidad o eficiencia de los servicios prestados. ${ }^{7}$ Sin este ajuste los hospitales donde se atienden pacientes con mayor riesgo de complicaciones están claramente en desventaja, al menos dos consecuencias podrían derivarse de la publicación de tasas no ajustadas: 1 ) se corre el riesgo de ser injustos en las evaluaciones del trabajo médico y hospitalario, 2) se propicia que el personal de la sala que trabaja directamente con el paciente acelere indebidamente las altas de los pacientes o realice alguna otra maniobra para reducir artificialmente el valor del indicador.

La necesidad de los ajustes es pues clara, no obstante, no todos los países exhiben datos ajustados de sus indicadores de resultados y no existe un consenso general sobre la manera en que ha de realizarse tal ajuste. En Cuba no se reportan tasas de resultados hospitalarios en los anuarios estadísticos actuales pero en las reuniones mensuales que se realizan en los hospitales para evaluar todo el trabajo hospitalario no es aún una norma utilizar indicadores ajustados. En el presente trabajo se recorren algunos conceptos necesarios para realizar el ajuste de riesgo que mejore la validez de las comparaciones del desempeño de servicios hospitalarios en tiempo y espacio. La idea es responder, de manera clara, a la pregunta: ¿Cómo realizar el ajuste de riesgo en los indicadores de resultados de la atención hospitalaria?

La respuesta a esta pregunta no resulta sencilla, algo fácilmente comprensible si se considera que la variedad de indicadores es muy amplia. I ezzoni, en un libro publicado en 1997, describe con amplitud diferentes métodos y sistemas que han sido utilizados para estos ajustes en las comparaciones de resultados. ${ }^{13}$ Este trabajo pretende recorrer los diferentes aspectos del problema sin adentrarse en vericuetos técnicos pero con suficiente amplitud para que el lector pueda emplear herramientas que lo ayuden a completar el conocimiento y aplicar ajustes de riesgo cuando sea necesario. I gualmente se pretende introducir esta inquietud en el escenario de la salud pública cubana.

\section{EL PLANTEAMI ENTO DEL PROBLeMA}


Todos los indicadores de resultados tienen una relación obvia con el tipo de pacientes atendidos en el período evaluado, esto equivale a plantear que el valor que tomen depende de una mezcla de factores: la efectividad del tratamiento, los factores de riesgo (del resultado en cuestión, muerte complicaciones, entre otros) del paciente que se imbrican con la efectividad del tratamiento, la calidad de la atención recibida (en toda su amplitud), y un componente aleatorio (siempre presente en la medición de aspectos relacionados con la actividad humana).

Remedando la notación matemática, con fines didácticos, se podría plantear la ecuación siguiente:

Resultados $=$ Efectividad del tratamiento $+\mathrm{FR}+$ Calidad atención + Componente aleatorio

Si se considera, como es común, la efectividad del tratamiento como parte de la calidad de la atención, el control de los factores de riesgo del paciente permite aislar el efecto de la calidad, puesto que el componente aleatorio se inquiere con métodos estadísticos. Este razonamiento parece sencillo pero su aplicación implica dificultades prácticas que se pueden resumir en dos: la determinación de las variables necesarias para los ajustes (lo que se llama factores de riesgo) y los métodos para lograr el ajuste adecuado.

\section{VARI ABLES PARA LOS AJ USTES}

De lo anterior se puede resumir que el riesgo inherente a las características previas del paciente, como la edad, el sexo, el diagnóstico principal (al ingreso), la gravedad de la enfermedad que constituye el diagnóstico principal, las enfermedades asociadas, los factores psicosociales y culturales, las características socioeconómicas y hasta las preferencias por cuidados específicos, teniendo en cuenta el papel del azar, más allá de la efectividad y eficiencia del proveedor, conducirán a diferentes resultados. ${ }^{12-19}$ En el lenguaje de la estadística, estas características indicadoras de riesgo de un resultado dado, se denominan variables. Algunas de las variables más utilizadas para los ajustes se presentan y comentan a continuación.

Una variable o factor de riesgo de particular importancia ha sido la llamada "gravedad de la enfermedad" (en inglés: illness severity). Se trata de un concepto que engloba distintos atributos de los pacientes y su enfermedad que pueden por separado considerarse factores de riesgo, como se ha mencionado antes. ${ }^{13} \mathrm{En}$ virtud de ello y en un afán parsimonioso y simplificador, han sido construidos índices de gravedad (generales y particulares para distintos servicios hospitalarios) que intentan evaluar de forma cuantificada la gravedad de los pacientes. Entre los primeros se encuentra el Indice de Horn ${ }^{20,21}$ y el índice propuesto por Jiménez ${ }^{22,23}$ para el escenario cubano. Entre los segundos, pueden señalarse como ejemplos el sistema APACHE (Acute Physiology and Chronic Health Evaluation), el MPM (Mortality Prediction Model) y el SAPS (Simplified Acute Physiology Score) que constituyen índices de gravedad para pacientes ingresados en una unidad de cuidados intensivos. Todos estos han sido utilizados para ajustes en la evaluación de la calidad y eficiencia de la gestión hospitalaria. ${ }^{24,25}$

Otra variable de interés especial en este recuento es el diagnóstico principal o enfermedad que da lugar al ingreso del paciente hospitalizado. En otras palabras, el diagnóstico principal de un paciente hospitalizado es aquel que, después de 
transcurrido esencialmente el proceso de hospitalización, se reconoce como la causa que dio lugar al ingreso amén de la posible presencia de otras enfermedades en el mismo paciente. ${ }^{13-15}$ Es conocido además que la gravedad puede diferir considerablemente entre categorías diagnósticas y agrupamientos diagnósticos, como argumentan Travassos y otros, ${ }^{16}$ de modo que tanto gravedad como diagnóstico, y eventualmente su interacción, deben considerarse para el ajuste de riesgo.

Las enfermedades asociadas (en inglés, comorbidity) constituyen otro factor de riesgo importante en los pacientes hospitalizados y muchas veces se incluyen en los índices de gravedad. Estas suelen además interactuar con el diagnóstico principal pues se conoce que ciertas combinaciones de condiciones morbosas presentan mayor riesgo de resultados negativos que otras. ${ }^{13,16,17}$

La edad también ha sido utilizada como una de las variables para el ajuste de riesgo pues se asocia a otras características del paciente como el propio diagnóstico y las enfermedades asociadas pero también suele tener una relación independiente con los peores resultados (muerte, complicaciones, duración de la estadía hospitalaria) ya que los ancianos pueden requerir mayores períodos de recuperación y tener mayor riesgo de complicaciones. ${ }^{13,16,26}$ I ezzoni ${ }^{27}$ señala que la edad puede tener un efecto independiente de otros atributos del paciente en el riesgo de morir.

El sexo es con frecuencia utilizado para los ajustes de las tasas de mortalidad hospitalaria, es una variable con menor influencia general en el riesgo de morir que la edad pero para condiciones específicas, como cirugía coronaria o infarto agudo del miocardio, el riesgo de morir difiere claramente entre hombres y mujeres. ${ }^{13,18,19}$ Algunos autores han destacado que la edad y el sexo tienen la ventaja de ser variables simples y directas con buena validez y alta confiabilidad en su registro. ${ }^{16}$

El tipo de admisión es una variable también considerada influyente sobre los resultados. Los pacientes con admisión electiva en general presentan mejor pronóstico que los admitidos por emergencia, algo muy evidente en los servicios quirúrgicos donde la preparación previa, sólo posible en operaciones electivas, resulta de importancia en el pronóstico del paciente. En el caso de que un paciente ingrese como traslado de otro hospital se generan situaciones que pueden influir de una manera $u$ otra sobre los resultados, los pacientes provenientes de hospitales concebidos para enfermedades crónicas suelen ser casos graves o terminales y por tanto tienen mayor riesgo de morir pero puede darse el caso contrario. ${ }^{16}$

Algunos autores han identificado otras variables que influyen sobre los resultados en los pacientes y que deberían incorporarse a los ajustes, por ejemplo el estado funcional, (capacidad de realizar la actividades de la vida diaria, limitaciones sensoriales, físicas y motoras) que no solamente está asociado a un gran consumo de recursos de enfermería, sino directamente al pronostico, el estado psicológico, cognitivo y psicosocial, que requiere de la realización de costosas entrevistas, las características socio-culturales como nivel económico, nivel de estudios, raza, alcoholismo, toxicomanías, y hábitos de alimentación, la calidad de vida previa, las actitudes y las preferencias del paciente (por ejemplo, rechazo a cuidados agresivos). ${ }^{12,13,16,27}$

En algunos países el nivel socio-económico ha sido señalado como una característica que influye sobre los resultados, aunque se plantea que el ajuste de indicadores por factores socioeconómicos tiene que considerar la posibilidad de que los pacientes con un nivel socioeconómico menos elevado reciban una atención de 
menor calidad, ${ }^{16,28,29}$ o sea, se puede producir una interacción entre la variable considerada y la calidad de la atención que repercute sobre la evaluación de los resultados. En el caso de la mortalidad hospitalaria por ejemplo, una asociación entre la muerte en el hospital y la clase social del paciente puede ser resultado de iniquidades en el cuidado de individuos socialmente distintos, en tal caso esta variable no debía incorporarse en el ajuste del riesgo de morir pues podría anularse su efecto sobre las tasas de mortalidad y no identificarse un problema que realmente se debe a la calidad de la atención. La interacción entre el nivel socioeconómico del paciente y el trato que este recibe da lugar a las llamadas disparidades o iniquidades en el uso de los servicios de salud y ha sido abordado repetidas veces por distintos autores. ${ }^{30,31}$

La distancia entre el hospital y la vivienda del paciente ha sido incluida en ajustes de riesgo, en particular cuando la estadía es el resultado evaluado. La forma de incluirla varía, algunos autores han utilizado una medida métrica de distancia, mientras que otros han incluido variables ordinales que dan cuenta del grado de lejanía que separa la vivienda del paciente del hospital. ${ }^{32-34}$ La justificación de incluir esta variable como factor de riesgo descansa en que el médico de asistencia suele mostrar cierta resistencia a dar el alta tempranamente a pacientes que tiene más dificultades para regresar, en caso necesario. No se tiene una referencia reciente de inclusión de esta variable en ajustes de riesgo pero, la justificación para incluirla continúa siendo válida.

En los servicios que atienden a pacientes graves, como las unidades de cuidados intensivos, la estabilidad clínica, que incluye una serie de variables que reflejan el estado fisiológico del paciente, influye directamente sobre el resultado principal que suele evaluarse en estos servicios, la mortalidad. Se incluyen aquí los signos vitales ( frecuencia cardiaca y respiratoria, presión arterial), el equilibrio bioquímico ( $\mathrm{K}^{+}$, $\mathrm{Na}^{+}$, creatinina), los parámetros hematológicos, los gases sanguíneos, el examen neurológico y el nivel de conciencia, variables que deben incorporarse a los ajustes de la mortalidad. ${ }^{13,16,35}$ Varios de los índices de gravedad, desarrollados para los pacientes graves, que se han utilizado en el ajuste de riesgo, contemplan estas mediciones. ${ }^{36}$

\section{LOS MÉTODOS PARA LOS AJ USTES. ¿SISTEMAS DE CLASI FI CACIÓN O MODELOS DE REGRESIÓN?}

La forma en que debe realizarse el ajuste transita por la respuesta a esta pregunta. Está claro que el ajuste conlleva estratificación, es decir, lo que se persigue es comparar dentro de grupos "comparables" ya que una comparación con fines valorativos que no respete este principio (grupos iguales en todo menos en la variable que se está evaluando) pierde validez, pero la introducción de métodos estadísticos multivariados permite, en cierta forma, obviar la formación explícita de los grupos (o estratos). De modo que aparece aquí una disyuntiva para los evaluadores: 1) conformar grupos de pacientes homogéneos en cuanto a características que influyan sobre la variable de resultados en cuestión y comparar entonces dentro de estos grupos o 2) aplicar las técnicas multivariadas que permiten de forma estadística realizar comparaciones "ajustadas."

\section{Estratificación para los ajustes}

La opción de formar grupos o estratos homogéneos de pacientes es, sin dudas, la más sencilla forma de ajuste y quizás la más extendida en la práctica. El ejemplo 
más relevante y elocuente de los sistemas de clasificación de pacientes lo brindan los Grupos de Diagnósticos Relacionados (GDR) (del inglés, Diagnosis Related Groups o DRG) que parece constituir el sistema de clasificación más ampliamente utilizado en el mundo. ${ }^{37,38}$ Este sistema de clasificación fue desarrollado a principios de la década de 1970 por Fetter y otros en la Universidad de Yale en los Estados Unidos. ${ }^{39}$ Medicare, la compañía de seguros más grande de los Estados Unidos en esa época, promovió el desarrollo estos grupos con la idea de introducir el llamado "pago prospectivo" (en inglés, prospective payment) y reducir así sus crecientes gastos por concepto de pago de seguros de salud a los hospitales que atendían a sus pacientes. Se crearon grupos de diagnósticos homogéneos en cuanto a consumo de recursos (a partir de la revisión de un millón de historias clínicas) de modo que la compañía paga por los pacientes dentro de cada grupo una cantidad fija de dinero con independencia del gasto real en que este incurra. Estos grupos homogéneos resultaron ser la base del sistema de pago prospectivo pero también comenzaron a constituir grupos propios para el "ajuste de riesgo" ya que, en general, la homogeneidad en cuanto a consumo de recursos resultó en homogeneidad en cuanto a gravedad de los pacientes incluidos. Los GDR han sido usados ampliamente como una herramienta para clasificar a los pacientes en grupos sobre la base de los recursos que se espera consuman durante la hospitalización y como base para el ajuste de riesgo. ${ }^{40}$ Algunos ejemplos de GDR se muestran en el recuadro.

Recuadro. Ejemplos de Grupos de Diagnósticos Relacionados

\begin{tabular}{|l|l|}
\hline GDR & Descripción \\
\hline 1 & Craneotomía edad $>17$ excepto por trauma \\
\hline 2 & Craneotomía por trauma edad > 17 \\
\hline 12 & Trastornos degenerativos de sistema nervioso \\
\hline 13 & Esclerosis múltiple y ataxia cerebelosa \\
\hline 14 & $\begin{array}{l}\text { Trastornos cerebro vasculares específicos excepto } \\
\text { accidente isquémico transitorio y hemorragia intracraneal }\end{array}$ \\
\hline 15 & $\begin{array}{l}\text { Accidente isquémico transitorio y oclusiones } \\
\text { precerebrales }\end{array}$ \\
\hline 87 & Edema pulmonar e insuficiência respiratoria \\
\hline 118 & $\begin{array}{l}\text { Revisión de marcapasos cardiaco sustitución de } \\
\text { generador }\end{array}$ \\
\hline
\end{tabular}

Otro sistema de clasificación conocido es el "Estadificación de la enfermedad" (del inglés Disease Staging). Este sistema fue propuesto por Gonella y otros para clasificar a los pacientes según la gravedad de la enfermedad en 4 niveles. ${ }^{41}$ Pero, a todas luces, este sistema ha sido utilizado mucho menos que el de los GDR.

Tanto en los GDR como en el sistema de Gonella y otros, la gravedad se le adjudica a un conjunto de características centradas en el diagnóstico; las categorías de gravedad se construyen independientemente de los pacientes involucrados en cada ajuste. En ambos casos se necesitan paneles de expertos para construir las combinaciones de los diagnósticos con otras características que definen los distintos grupos y grado de gravedad. Por tal motivo, ambos sistemas se han convertido en tecnología comercializada y los hospitales deben comprarlos para poder hacer uso de ellos. 
El ajuste con los sistemas de clasificación tiene la ventaja de que puede realizarse con los métodos tradicionales de ajuste de tasas, conocidos como método directo e indirecto ${ }^{7}$ pero, con cierta frecuencia, el ajuste sólo consiste en hacer comparaciones dentro de los grupos dados por los sistemas de clasificación.

Utilizar sistemas de clasificación significa por tanto, adherirse al método más simple de ajuste de riesgo y quizás por esto, resulta el método más aceptado. Sin embargo, hay dos objeciones para esta afirmación: 1) los grupos dentro de esos sistemas involucran más de una variable, casi siempre más de dos variables, de modo que la simplificación es sólo aparente y 2) al convertirse en sistemas comerciales, el evaluador se ve forzado a utilizarlos sin analizar el grado de ajuste que se logra ni saber a ciencia cierta cuales variables están siendo contempladas en el ajuste. Esta idea se desarrolla en el Informe Técnico de la Agencia de Evaluación de Tecnologías Sanitarias (AETS) del Instituto de Salud "Carlos III" del Ministerio de Sanidad y Consumo del Estado Español publicado en septiembre de 2007 donde se hace una evaluación de los sistemas de ajuste de riesgo existentes en la actualidad para la evaluación de servicios de salud. ${ }^{42}$

\section{Métodos multivariados: modelos de regresión}

Los modelos de regresión constituyen la segunda variante para el ajuste de riesgo. Se trata de funciones matemáticas donde se distingue una variable de respuesta (o dependiente) y varias variables explicativas (o independientes). Se supone que la función (de las variables explicativas) pueda explicar la mayor parte de la variación de la variable de respuesta y se demuestra que dichos modelos son capaces de "aislar" el efecto (o la relación) que cada variable explicativa tiene sobre la variable de respuesta. Esto permite alcanzar lo que precisamente se persigue con los ajustes, el control de los efectos de variables potencialmente de confusión sobre la relación que supuestamente se está midiendo.

Los dos modelos de regresión con más utilidad en este ámbito son el modelo de regresión logística y el modelo de regresión múltiple.

Regresión logística. En la regresión logística la probabilidad de que ocurra determinado suceso se expresa en función de las variables consideradas influyentes. La ecuación que representa al modelo es la siguiente:

$$
\operatorname{Prob}(Y=1)=\frac{l}{l+\exp \left(-b_{0}-b_{1} X_{1}-\ldots-b_{n} X_{n}\right)}
$$

donde: $Y$ representa a la variable de respuesta dicotómica $(Y=1$ si ocurre el suceso y $Y=0$ si no ocurre) y Prob $(Y=1)$ representa la probabilidad de que ocurra el suceso, $b_{0}$ : es el término independiente y $b_{1}, \ldots, b_{n}$ : son los coeficientes respectivos de las variables explicativas independientes $X_{i}$ que pueden ser cuantitativas o dicotómicas e incluso, mediante el empleo de cierto recurso estadístico (variables dummy) se permite incluir en la función variables cualitativas nominales $u$ ordinales. Una explicación detallada del modelo de regresión logística y sus aplicaciones puede encontrarse en diversas publicaciones ${ }^{43,44}$ y ha sido muy utilizado para calcular indicadores ajustados por riesgo. ${ }^{45-47}$

Con la regresión logística se estiman las "tasas esperadas" de determinado resultado bajo ciertas condiciones (dadas por los valores de las $X_{i}$ ) y esas tasas se podrán comparar con las observadas. De esta manera se están controlando los 
factores de confusión identificados en la comparación. Por ejemplo, si tenemos una función de regresión logística que explica bien la probabilidad de morir a partir de la edad, la gravedad y el diagnóstico principal de los pacientes, se puede estimar la tasa de mortalidad esperada en un servicio en determinado período de tiempo (dado el tipo de pacientes que ingresan en ese período; el número esperado de muertes es la suma de las probabilidades de morir estimadas por la función para cada paciente) y compararla con la observada en ese período. Si la tasa observada es mucho mayor que la esperada, la diferencia podrá atribuirse a problemas de calidad en la atención ya que otros factores que pudieran explicar la mortalidad están presuntamente controlados.

En la tabla 1 se muestra un ejemplo del uso de la regresión logística para ajustar el riesgo de morir según algunas variables que fue efectuado en el Hospital Clínico Quirúrgico "Hermanos Ameijeiras" durante el año 2006 para buscar un modelo predictivo para la mortalidad en una unidad de cuidados intensivos.

La variable dependiente dicotómica fue la mortalidad (o estado al egreso: fallecido o vivo) y el resto fueron las variables explicativas introducidas en el modelo. Con la función encontrada se calculan las probabilidades de muerte de cada paciente y la suma de todas ellas sería el número de defunciones esperadas.

Tabla 1. Estimación de la función de regresión logística. Unidad de cuidados intensivos. Hospital Clínico Quirúrgico "Hermanos Ameijeiras" 2006

\begin{tabular}{|c|c|c|c|c|c|c|}
\hline \multirow[t]{2}{*}{ Variables } & \multirow[t]{2}{*}{ B } & \multirow[t]{2}{*}{$\mathrm{EE}$} & \multirow[t]{2}{*}{ Sig. } & \multirow[t]{2}{*}{$\begin{array}{l}\operatorname{Exp} \\
\text { (B) }\end{array}$} & \multicolumn{2}{|c|}{$\begin{array}{c}\text { Intervalo de } \\
\text { confianza } \\
95 \% \operatorname{Exp}(B)\end{array}$} \\
\hline & & & & & $\begin{array}{l}\text { Límite } \\
\text { inferior }\end{array}$ & $\begin{array}{l}\text { Límite } \\
\text { superior }\end{array}$ \\
\hline Constante & $4, \overline{378}$ & 0,741 & 0,000 & 0,013 & & \\
\hline Edad & 0,017 & 0,10 & 0,093 & 1,018 & 0,997 & 1,038 \\
\hline APACHE & 0,099 & 0,25 & 0,000 & 1,091 & 1,038 & 1,146 \\
\hline \multicolumn{7}{|c|}{ Grupos diagnósticos (variables dummy) ${ }^{2}$} \\
\hline $\begin{array}{l}\text { Grupo 2: } \\
\text { Ginecoobstétrico, } \\
\text { Endocrino- } \\
\text { metabólico y } \\
\text { renal }\end{array}$ & $2, \overline{0} 27$ & 1,087 & 0,062 & 0,132 & 0,016 & 1,110 \\
\hline $\begin{array}{l}\text { Grupo 3: } \\
\text { Digestivo, } \\
\text { neurológico y } \\
\text { otros, }\end{array}$ & 0,911 & 0,389 & 0,019 & 2,486 & 1,059 & 5,330 \\
\hline $\begin{array}{l}\text { Grupo 4: } \\
\text { Hemolinfopoyético }\end{array}$ & 3,737 & 1,187 & 0,002 & 41,978 & 4,099 & 429,931 \\
\hline Estado quirúrgico & 0,373 & 0,399 & 0,350 & 1,452 & 0,665 & 3,170 \\
\hline
\end{tabular}

${ }^{1}$ La función se estimó con 260 pacientes, ${ }^{2}$ Variables ficticias (en inglés: dummy variables) que se utilizan para introducir en la función variables de carácter 
cualitativo nominal. Se elige un grupo de referencia que en este caso es el grupo 1

(Cardiovascular, espiratorio y SOMA).

Fuente: López Lamezón S. Modelo predictivo de la mortalidad en la unidad de cuidados intensivos del hospital "Hermanos Ameijeiras". 2002-2004. Trabajo para optar por el título de Especialista de I Grado en Bioestadística.

Regresión lineal múltiple. La regresión lineal múltiple permite un análisis similar para situaciones donde la variable de respuesta es cuantitativa y por esto se ha utilizado en los ajustes para evaluar la estadía como indicador de resultados.

En ese caso, la ecuación que representa al modelo: ${ }^{48}$

$$
Y_{i}=b_{0}+b_{1} X_{1 i}+b_{2} X_{2 i}+\ldots \ldots+b_{n} X_{n i}+e_{i}
$$

es similar a la vista antes, sólo que aquí Y representa a una variable de respuesta cuantitativa.

Se ha utilizado para analizar la estadía "ajustando" por otras variables. Con las variables explicativas adecuadas (edad, gravedad, diagnóstico y otras) se puede estimar la estadía esperada para cada paciente. Las diferencias con la estadía observada pueden atribuírsele a problemas en la atención recibida. Del mismo modo es posible estimar el promedio ajustado de estadía y compararlo con el observado. Jiménez y otros desarrollaron y evaluaron en Cuba un sistema de este tipo en los servicios de medicina interna, cirugía general y psiquiatría en un hospital clínico quirúrgico. ${ }^{49-51}$

En ambos casos el ajuste consiste en "controlar" las variables o características del paciente que también pueden explicar el suceso (muerte, estadía u otro) y aislar así los posibles problemas en la atención.

Dos dificultades principales se señalan para la aplicación práctica de estos modelos: 1) la necesidad de recoger una serie de datos sobre el paciente no son extraídos rutinariamente de la historia clínica y 2) la interpretación de los ajustes, que demanda conocimientos de estadística relativamente avanzados. La primera dificultad demanda un sistema de información más amplio y dinámico que el que existe actualmente en muchos hospitales y la segunda, mayor capacitación para el personal de dirección y el de registros médicos de los hospitales. Actualmente, con el uso de la computación, que facilita el manejo del sistema de información y los cálculos que demandan, estos modelos resultan realmente atractivos.

Un ejemplo del uso reciente de un modelo de regresión lineal múltiple para el ajuste de riesgo es un estudio realizado en el servicio de psiquiatría del Hospital Clínico Quirúrgico "Hermanos Ameijeiras". ${ }^{50}$ Primeramente se identificaron las variables que influían en la estadía hospitalaria mediante una ecuación de regresión múltiple (tabla 2). Posteriormente se calculó la diferencia promedio entre la estadía observada y la esperada, estimada esta última con la función de regresión múltiple. Se pudo comprobar que los pacientes con valores más altos de esta diferencia tenían los problemas más graves de ineficiencia en el cuidado de los pacientes.

Tabla 2. Estimación de la función de regresión múltiple. Servicio de psiquiatría. Hospital Clínico Quirúrgico "Hermanos Ameijeiras".1999 


\begin{tabular}{|c|c|c|c|c|}
\hline \multirow{2}{*}{ Variables } & \multicolumn{2}{|c|}{ Coeficientes } & \multicolumn{2}{|c|}{$\begin{array}{l}\text { Coeficientes } \\
\text { estandarizados }\end{array}$} \\
\hline & B & $\begin{array}{l}\text { Error } \\
\mathrm{E}\end{array}$ & Beta & Sig. \\
\hline Constante & 2,358 & 0,189 & & 0,000 \\
\hline $\begin{array}{l}\text { Trastornos del estado de } \\
\text { ánimo }^{1}\end{array}$ & $-0,085$ & 0,086 & $-0,103$ & 0,325 \\
\hline $\begin{array}{l}\text { Esquizofrenia, trastornos } \\
\text { delirantes }^{1}\end{array}$ & $-0,179$ & 0,102 & $-0,165$ & 0,082 \\
\hline $\begin{array}{l}\text { Trastornos de } \\
\text { personalidad }^{1}\end{array}$ & $-0,238$ & 0,092 & $-0,236$ & 0,011 \\
\hline Trastornos adaptativos $^{1}$ & $-0,157$ & 0,102 & $-0,141$ & 0,125 \\
\hline Edad & 0,007 & 0,002 & 0,254 & 0,002 \\
\hline $\operatorname{Sexo}(1 \mathrm{M}, 2 \mathrm{~F})$ & 0,029 & 0,056 & 0,037 & 0,605 \\
\hline $\begin{array}{l}\text { Centro Habana y Habana } \\
\text { Vieja }^{2}\end{array}$ & $-0,017$ & 0,082 & $-0,020$ & 0,836 \\
\hline $\begin{array}{l}\text { Otros municipios en } \\
\text { Ciudad Habana }^{2}\end{array}$ & $-0,074$ & 0,077 & $-0,093$ & 0,339 \\
\hline Provincia Habana ${ }^{2}$ & 0,097 & 0,100 & 0,077 & 0,332 \\
\hline Estado civil & 0,093 & 0,055 & 0,118 & 0,095 \\
\hline Ninguno & 0,051 & 0,073 & 0,058 & 0,481 \\
\hline Drogas antidepresivas ${ }^{3}$ & 0,109 & 0,071 & 0,132 & 0,125 \\
\hline $\begin{array}{l}\text { Terapia } \\
\text { electroconvulsivante }\end{array}$ & 0,267 & 0,088 & 0,215 & 0,003 \\
\hline Ninguno & $-0,030$ & 0,070 & $-0,037$ & 0,674 \\
\hline Enfermedades sistémicas ${ }^{4}$ & 0,010 & 0,076 & 0,012 & 0,891 \\
\hline Respuesta al tratamiento & 0,230 & 0,064 & 0,246 & 0,000 \\
\hline \multicolumn{5}{|l|}{ Síntomas ${ }^{5}$} \\
\hline Factor 1 & 0,069 & 0,031 & 0,174 & 0,025 \\
\hline Factor 2 & 0,051 & 0,027 & 0,128 & 0,059 \\
\hline Factor 3 & 0,080 & 0,026 & 0,203 & 0,003 \\
\hline Factor 4 & $-0,019$ & 0,026 & $-0,048$ & 0,461 \\
\hline Factor 5 & $\mid-0,013$ & 0,026 & $-0,032$ & 0,626 \\
\hline Factor 6 & 0,018 & 0,027 & 0,046 & 0,504 \\
\hline Factor 7 & 0,0009 & 0,026 & 0,002 & 0,974 \\
\hline Factor 8 & 0,029 & 0,027 & 0,074 & 0,828 \\
\hline
\end{tabular}

${ }^{1}$ Dummy para el diagnóstico. Categoría de referencia: resto de los diagnósticos, ${ }^{2}$ Dummy para el lugar de residencia. Categoría de referencia: otra provincia, ${ }^{3}$ Dummy para medicamentos. Categoría de referencia: antipsicóticos,

${ }^{4}$ Dummy para enfermedades asociadas. Categoría de referencia: otras enfermedades asociadas, ${ }^{5}$ valor del factor para uno de los 8 componentes principales de los síntomas.

Fuente: Jiménez RE, Lam RM, Marot M, Delgado A. Observed-predicted length of 
stay for an acute psychiatric department, as an indicator of inpatient care inefficiencies. Retrospective case-series study. BMC Health Serv Res. 2004; 4:4.

Por último, cabe destacar la necesidad de parsimonia en la búsqueda de las variables y métodos para los ajustes. Cada paciente es único, como afirma Rodríguez, ${ }^{52}$ lo que sugiere que las variables que pueden influir sobre los resultados de la atención son una cantidad imposible de manejar; el problema estriba entonces en buscar la menor cantidad de variables que permita una valoración acertada. Este es básicamente el reto que tienen que enfrentar epidemiólogos y administradores en la implementación de ajustes adecuados para sus valoraciones. Afortunadamente, se han ido conociendo las variables más importantes y los métodos mejores para incluirlas. En particular las técnicas estadísticas que se emplean para aplicar los modelos de regresión brindan herramientas para selección de variables que, unidas al sentido común, permiten buscar el balance óptimo entre necesidad y posibilidad en este sentido.

Un estudio reciente realizado en España por la Agencia de Evaluación de Tecnologías Sanitarias, incursiona, a través de una revisión sistemática de la literatura, en las variantes utilizadas actualmente para los métodos de ajuste de riesgo, su utilidad y necesidad actual y el uso de modelos estadísticos que contemplan distintas variables en la búsqueda del balance necesario entre parsimonia y eficacia predictiva versus los sistemas comerciales. ${ }^{42}$

En Cuba, la compra de sistemas de clasificación significaría un gasto elevado para el sistema público de salud. La opción de los ajustes por modelos de regresión es más acorde con la situación socioeconómica actual, el principal obstáculo para su implantación radica en la falta de información sobre variables importantes que deben utilizarse en los ajustes. Ampliar y perfeccionar el sistema de información existente para recoger los datos necesarios para el ajuste de riesgo es el camino a seguir en el perfeccionamiento de los indicadores de desempeño hospitalario. El actual empeño del Ministerio de Salud Pública de Cuba por lograr la informatización de la gestión hospitalaria constituye un sustrato excelente para esta meta.

A modo de resumen se enfatiza en la necesidad de evaluar de forma continua el desempeño de los hospitales cubanos utilizando para ello los métodos más modernos que contemplan la introducción del ajuste de riesgo para las comparaciones temporales y espaciales que entraña la propia evaluación. Para esto es necesario la modernización y consiguiente automatización de los actuales sistemas de información y la capacitación adecuada de los administradores, directores y personal de los departamentos de registros médicos hospitalarios.

\section{REFERENCI AS BI BLI OGRÁFI CAS}

1. Ríos Massabot NE. Interpretación de los indicadores que miden la utilización de las camas hospitalarias. Rev Cubana Adm Salud. 1987; 4:47-58.

2. . Los indicadores hospitalarios de uso más frecuentes y su utilización en la administración de las instituciones de este tipo. Selección de artículos. La Habana: ISCM-H, Facultad de Salud Pública; 1987. 
3. European Observatory on Health Care Systems. WHO Regional Office for Europe [sitio en Internet]. [citado 26 mar 2008]. Disponible en: http:// www.observatory. dk

4. Corella JM. La Gestión de servicios de salud. Cap 11. Madrid: Díaz de Santos; 1996.

5. Gilmore CM, De Moraes H. Manual de gerencia de la calidad. Washington, D.C.: OPS PALTEX; 1996.

6. Secretaría de Salud. México. Observatorio del Desempeño Hospitalario 2004. 2005 [sitio en Internet]. [citado 2 Ago 2007]. Disponible en:

http://evaluacion.salud.gob.mx/publicaciones/odh/odh2004/odh2004.pdf

7. Jiménez Paneque RE. Indicadores de calidad y eficiencia de los servicios hospitalarios: una mirada actual. Rev Cubana Salud Pública. 2004; 30(1): 17-36.

8. Iezzoni LI. Risk and Outcomes. En: Iezzoni LI, editora. Risk adjustment for measuring healthcare outcomes. 2nd ed. Chicago, Illinois: Health Administration Press; 1997. p.1-41.

9. Risk Adjustment Current Health Policy Initiatives. En: Iezzoni LI, editora. Risk adjustment for measuring healthcare outcomes. 2nd ed .Chicago, Illinois: Health Administration Press; 1997.p. 517-95.

10. $1997 ; 278(19): 325-43$

The risk of risks adjustment (special communications). JAMA.

11. Response to letter by William Farr. Medical Times and Gazette. 13 February 1864: 187-8.

12. Iezzoni LI. 100 Apples Divided by 15 Red Herrings: A Cautionary Tale from the Mid-19th Century on Comparing Hospital Mortality Rates. Ann Internal Med. 1996; 124(15): 1079-85.

13. Peiró S. Evaluación de la calidad a partir del Conjunto Mínimo de Datos Básicos (CMBD) al alta hospitalaria. Instituto de Investigación en Servicios de Salud [sitio en Internet]. 1998 [ citado 2 Ago 2007]. Disponible en:

http://neurologia. rediris. es/congreso-1/conferencias/asistencia-2.html

14. Orchard C. Comparing healthcare outcomes. BMJ . 1994; 308: 1493-6.

15. Zhang W, Ayanian JZ, Zaslavsky AM. Patient characteristics and hospital quality for colorectal cancer surgery. Int J Qual Health Care. 2007;19(1):11-20.

16. Travassos C, Carvalho de Noronha J, Martins M. Mortalidade hospitalar como indicador de qualidade: uma revisão Ciênc. Saúde Coletiva. 1999;4(2):367-81.

17. DesHarnais SI, Chesney JD, Wroblewski RT, Fleming ST, McMahon LF J r. The risk-adjusted mortality index a new measure of hospital performance. Med Care. 1988; 26(12): 1129-48. 
18. Hannan EL, Kilburn H Jr, Racz M, Shields E, Chassin MR. Improving the outcomes of coronary artery bypass surgery in New York State. JAMA.

1994; 271: 761-6.

19. Fowler RA, Sabur N, Li P, Juurlink DN, Pinto R, Hladunewich MA, et al. Sex- and age-based differences in the delivery and outcomes of critical care. CMAJ.

2007; 177(12): 1513-9.

20. Horn SD, Sharkey PD, Bertran DA. Measuring severity of illness. Homogeneous case mix groups. Med Care. 1983;21(1):14-30.

21. Horn SD, Horn RA. Reliability and validity of the severity of illness index. Med Care. 1986; 24(2): 159-78.

22. Jiménez RE, Vázquez J, Fariñas H. Construcción y validación de un índice de gravedad para pacientes hospitalizados en áreas clínicas. Gac Sanit, Xunta de Galicia. 1997; 1:122-30.

23. Jiménez RE, Domínguez E, Fariñas $H$, Fuentes $E$. Construcción y validación de un índice de gravedad para pacientes hospitalizados en áreas quirúrgicas. Rev Cubana Salud Pública. 1999;25(2): 154-66.

24. Knaus WA, Zimmerman JE, Wagner DP, Draper EA, Lawrence DE. APACHE: acute physiology and chronic health evaluation: a phisiologically based classification system. Crit Care Med. 1981; 9:591-7.

25. Tees D, Lemeshow S, Haris D. Mortality prediction model (MPM) for ICU patients. Probl Crit Care. 1989; 3(7):585-90.

26. Rômulo Cristovão de Souza R, Sobrino Pinheiro R, Medina Coeli C, Rochel de Camargo K. The Charlson comorbidity index $(\mathrm{CCl})$ for adjustment of hip fracture mortality in the elderly: analysis of the importance of recording secondary diagnoses. Cad. Saúde Pública. 2008;24(2):315-22.

27. Daley J, Shwartz M. Developing Risk-Adjustment Methods. En: Iezzoni LI, editor. Risk adjustment for measuring healthcare outcomes. 2nd ed. Chicago, Illinois: Health AdministrationPress; 1997.p.279-329.

28. Frohlich $\mathrm{KL}$, Ross N, Richmon C. Health disparities in Canada today: some evidence and a theoretical framework. Health Policy. 2006; 79(2-3):132-43.

29. Bernheim SM, Ross JS, Krumholz HM, Bradley EH. Influence of patients' socioeconomic status on clinical management decisions: a qualitative study. Ann Fam Med. 2008; 6(1):53-9.

30. Robinson RG. Community Development Model for Public Health. Applications: Overview of a Model to Eliminate Population Disparities. Health Promotion Prac. $2005 ; 6(3): 338-46$.

31. Murray CJ L, Kulkarni SC, Michaud C, Tomijima N, Bulzacchelli MT, I andiorio TJ, et al. Eight Americas: Investigating Mortality Disparities across Races, Counties, and Race-Counties in the United States. PLoS Medicine. 2006;3(9): e260. 
32. J ones KR. Predicting hospital charge and stay variation. Med Care. 1985; 23(3) 220-35.

33. Vollertsen RS, Nobrega FT, Michet CJ J r, Hanson TJ, Naessens J M. Economic Outcome under Medicare Prospective Payment at a Tertiary-Care Institution: The Effects of Demographic, Clinical, and Logistic Factors on Duration of Hospital Stay and Past a Charge for Medical Back Problems. Mayo Clin Proc. 1988;63:583-91.

34. Berki SE, Ashcraft ML, Newbrander WC. Length of stay variations within ICD-8 Diagnosis-Related Groups. Med Care. 1984;22(2):126-42.

35. Iezzoni LI. Dimensions of risk. En: Iezzoni L, editor. Risk adjustment for measuring healthcare outcomes. 2nd ed. Chicago, Illinois: Health Administration Press; 1997.p.43-167.

36. Abizanda C. Comparación de APACHE II y SAPS como indicadores pronósticos. Med Intens. 1990; 14(6);259-63.

37. Noronha MF, Portela MC, Lebrão ML. Potential uses of AP-DRG to describe the health care profile in hospital units. Cad. Saúde Pública. 2004;20(2):242-55.

38. Moya del Madrigal L. Aplicación de los grupos de diagnósticos relacionados a la gestión del sistema nacional del servicios de la hospitalización de la CCSS. Rev Cienc Adm Financ Segur Soc. 1998; 6(2):13-33.

39. Fetter RB, Freeman JL, Mullin RL. DRGs: how they evolved and are changing the way hospitals are managed. Pathologist. 1985;39(6): 17-21.

40. Astier Peña MP, de Val Pardo I, Gost Garde J, Silvestre Busto MC, Larrayoz Dutrey M, Chivite Izco M, et al. Propuesta de indicadores para cuadros de mando de servicios médicos y quirúrgicos. Rev Adm Sanit. 2004;2(3):485-507.

41. Gonella JS, Louis DZ. Disease staging applications for utilization review and quality assurance. JAMA. 1984;251(5):637-44.

42. Palma Ruiz M, Sendra Gutiérrez J M, Sarría Santamera A. Sistemas de ajuste de riesgo en evaluación de Servicios de Salud. Madrid: Ministerio de Sanidad y Consumo;2007. [Agencia de Evaluación de Tecnologías Sanitarias, Instituto de Salud "Carlos III". Informe de Evaluación de Tecnologías Sanitarias № 51, Septiembre de 2007].

43. Silva LC. Excursión a la regresión logística en ciencias de la salud. Madrid: Díaz de Santos; 1995.

44. Molinero LM. La Regresión Logística (I) y (II) [monografía en Internet]. Enero 2001 [citado 15 Mar 2008]. Disponible en: http://www. seh-lelha.org/pdf/rlogis1.pdf

45. Domínguez L. Mortalidad y estancia hospitalaria ajustada por gravedad como indicadores de efectividad y eficiencia de la atención de pacientes en Unidades de Cuidados Intensivos. Med Intensiva. 2008; 32(1):8-14.

46. Cram P, Rosenthal GE, Vaughan MS. Cardiac Revascularization in Specialty and General Hospitals. N Engl J Med. 2005; 352: 1454-62. 
47. Hariharan S, Merritt-Charles L, Chen D. Risk-Adjusted Outcome Evaluation in a Multidisciplinary Intensive Care Unit. West Indian Med J . 2007;56(3):240-5.

48. Pértiga Díaz S, Pita Fernández S. Técnicas de regresión: Regresión Lineal Múltiple. Cad. Aten Primaria. 2000; 7:173-6.

49. Jiménez RE, López L, Domínguez D, Fariñas H. Difference between observed and predicted lenght of stay as indicator of patient care inefficiency. Internat J Quality Health Care. 1999; 11(5):375-84.

50. Jiménez RE, Lam RM, Marot M, Delgado A. Observed-predicted length of stay for an acute psychiatric department, as an indicator of inpatient care inefficiencies. Retrospective case-series study. BMC Health Serv Res. 2004; 4:4.

51. Jiménez Paneque RE, Gutierrez Rojas AR, Fariñas Seijas H, Suárez García N, Fuentes Valdés $E$. Variations in the postoperative length of stay according to patient characteristics in general surgery service. Gac Sanit. 1994;8(43): 180-8.

52. Rodríguez Rivera L. La clínica y su método. Reflexiones sobre dos épocas. Cap. 4. Madrid: Díaz de Santos; 1999.

Recibido: 21 de octubre de 2008.

Aprobado: 22 de noviembre de 2008.

Teddy Osmin Tamargo Barbeito. Hospital Clínico Quirúrgico "Hermanos Ameijeiras". La Habana, Cuba.

E-mail: teosmin@infomed.sld.cu 